\title{
The Mirror's Magic Sights: An Update on Mirror Symmetry
}

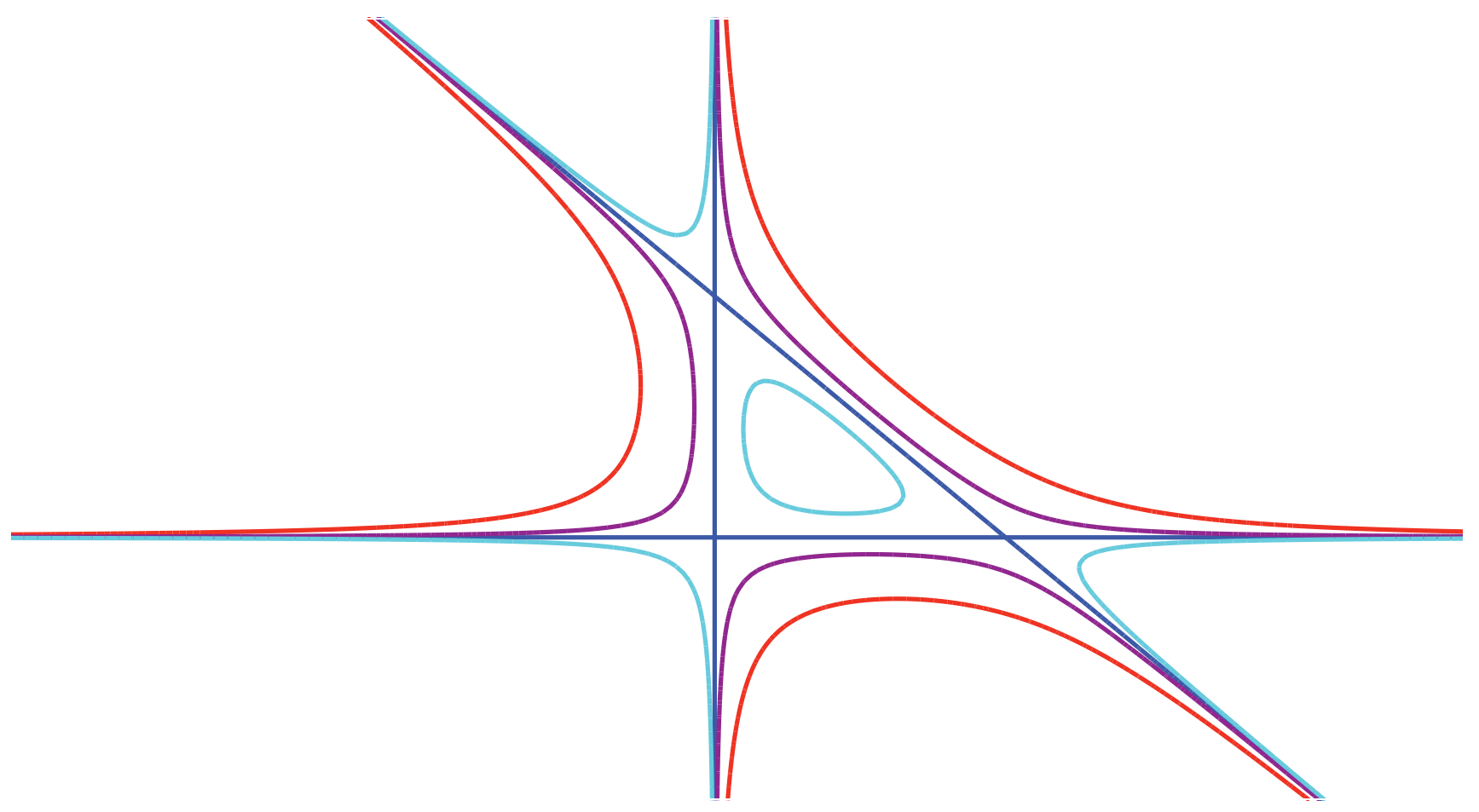

Timothy Perutz

But in her web she still delights

To weave the mirror's magic sights

Tennyson, The Lady of Shalott

\section{The Mirror Symmetry Mystery}

Origins. The 1980s and '90s saw an astonishing entanglement of research in geometry and mathematical physics. String theorists, developing their candidate for a quantum theory incorporating gravity, not only drew on state-ofthe-art mathematics, but introduced mathematical ideas of great power and prescience: none more so than mirror symmetry.

The author is an associate professor of mathematics at the University of Texas at Austin. His work is partially supported by NSF grant CAREER: 1455265. His email address is perutz@math . utexas . edu.

For permission to reprint this article, please contact:

reprint-permission@ams.org.

DOI: https://doi.org/10.1090/noti/1854
In a 1989 paper [13], Lerche, Vafa, and Warner studied the algebraic structure of 2-dimensional $N=2$ supersymmetric conformal field theories (SCFT). I will not define a 2dimensional $N=2$ SCFT, but only note that it is a type of quantum field theory-as such, involving operators on Hilbert spaces-in which the operators are associated with Riemann surfaces. The authors knew that a Calabi-Yau manifold gives rise to an $N=2$ SCFT, the Riemann surfaces being traced out by the motions and interactions of closed strings, i.e., loops, inside the manifold. In such a theory, they wrote,

there are four types of rings arising from the various combinations of chiral and anti-chiral, and left and right. We will denote these rings by $(a, c)$, $(a, a),(c, a),(a, c) . .$. There is a non-trivial relationship between $(c, c)$ and $(a, c)$. ... For superconformal models coming from compactification on Calabi-Yau manifolds, the $(c, c)$ ring becomes isomorphic to the structure of the cohomology ring of the manifold in the large radius limit. 

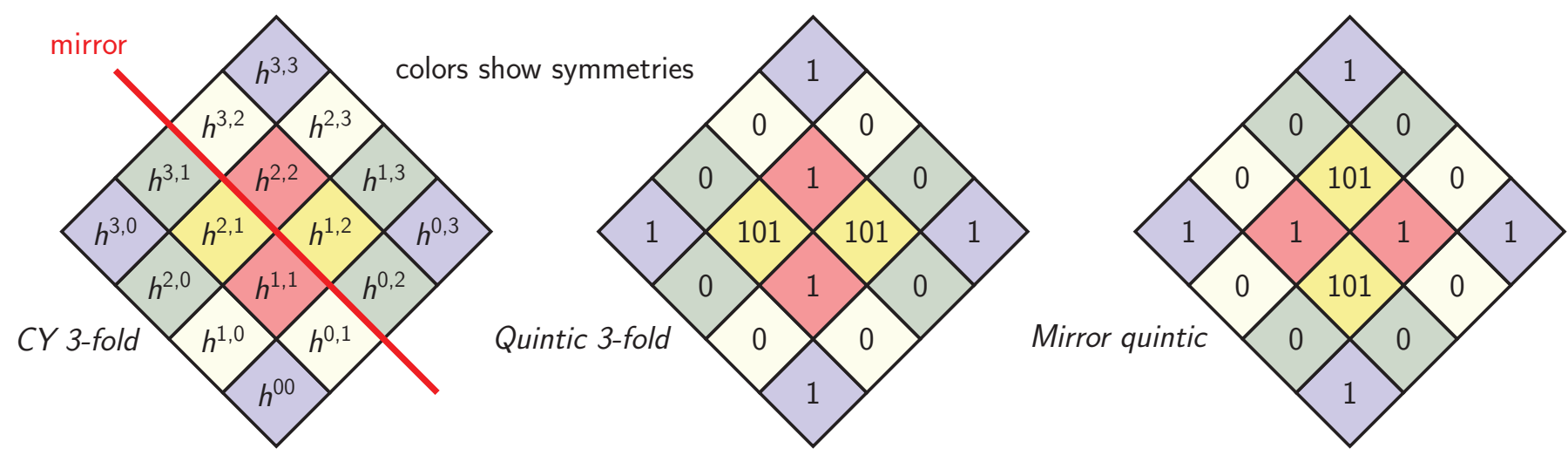

Figure 1. Mirrored Hodge diamonds for mirror pairs of $\mathrm{CY} 3$-folds.

... One possibility might be that [the Poincare series for the $(a, c)$ ring] is the Poincaré series for (a deformation of) the cohomology ring of another manifold. If so, there must be another manifold $\tilde{M}$ for which the Betti numbers satisfy $b_{p, q}^{M}=b_{d-p, q}^{\tilde{M}}$.

The possibility tentatively put forward in this passage ${ }^{1}$ was soon enunciated with greater precision and certitude, and named mirror symmetry $[9,19]$.

Basic explanations: A Kähler manifold $(M, \omega)$ is a complex manifold $M$, together with a Kähler form $\omega$ : a $C^{\infty}$ real 2-form-i.e., a skew-symmetric bilinear form on the tangent bundle $T M$-which is closed $(\mathrm{d} \omega=0)$, invariant under the complex structure, and positive on complex lines in $T M$. Being closed and non-degenerate, a Kähler form is an example of a symplectic form. Complex projective space $\mathbb{P}^{N}$ has a unique Kähler form (up to a positive scalar factor) that is invariant under the transitive action of the projective unitary group of $\mathbb{C}^{N+1}$; an embedding of $M$ into $\mathbb{P}^{N}$ determines a Kähler form on $M$ by restriction.

A Calabi-Yau (CY) manifold $(M, \omega, \Omega)$ is a compact Kähler manifold $(M, \omega)$ endowed with a holomorphic volume form $\Omega$. In local holomorphic coordinates $\left(z_{1}, \ldots, z_{d}\right)$, $\Omega=f(z) \mathrm{d} z_{1} \wedge \cdots \wedge \mathrm{d} z_{d}$, where $f$ is holomorphic and nowhere-vanishing. Examples:

- When $d=1$, the only CY manifolds are elliptic curves $\mathbb{C} /$ lattice; one can take $\omega=i \mathrm{~d} z \wedge \mathrm{d} \bar{z}$ and $\Omega=\mathrm{d} z$.

- CY hypersurfaces $M \subset \mathbb{P}^{d+1}$, cut out from projective space by a homogeneous polynomial of degree $d+2$. Elliptic curves arise as cubics in $\mathbb{P}^{2}$.

- Complex tori $\mathbb{C}^{d} /$ lattice.

The 'Betti numbers' $b_{p, q}^{M}$ in the quotation are really the Hodge numbers, $b_{p, q}^{M}=h^{p, q}(M):=\operatorname{dim}_{\mathbb{C}} H^{q}\left(M, \Omega_{M}^{p}\right)$ : $h^{p, q}$ is the vector-space dimension of the $q$ th cohomology of the sheaf $\Omega_{M}^{p}$ of holomorphic $p$-forms. The Betti number $b^{i}=\operatorname{dim}_{\mathbb{C}} H^{i}(M ; \mathbb{C})$, the dimension of the $i$ th singular cohomology, is the sum of the $h^{p, q}$ where $p+q=$

\footnotetext{
${ }^{1}$ L. Dixon reportedly also put forward this idea.
}

$i$. The 'Poincaré series' $P(t)$ of a graded ring is the generating function for the dimensions of its homogeneous parts, so for $H^{*}(M ; \mathbb{C})$ the Poincaré series is the polynomial $P(t)=\sum b^{i}(M) t^{i}$.

The term 'mirror symmetry' refers to a literal mirroring of Hodge diamonds expressed by the relation $h^{p, q}(M)=$ $h^{d-p, q}(\tilde{M})$ - the Hodge diamond is the conventional visualization of the array of Hodge numbers $h^{p, q}$ (Figure 1). But in retrospect, it seems mistaken to view that as a primary manifestation of mirror symmetry. I prefer to think of the term as a metaphor for the reciprocal relationship of $M$ to $\tilde{M}$-the mirror of the mirror is the original.

The $N=2$ SCFT which, string theorists argue, can be associated with a CY manifold $M$ is a type of sigma model: it is based on maps $\Sigma \rightarrow M$ where $\Sigma$ is a Riemann surface. There are two topological twists of the sigma model which are 2-dimensional topological field theories, called the A-model and the B-model. Formally they are on an equal footing, but their physical observables have quite different geometrical meanings, relating to holomorphic maps from Riemann surfaces to the CY in the A-model, and to period integrals of differential forms in the B-model. A statement of mirror symmetry, arising from string theory but congenial to mathematicians, is the following:

Mirror symmetry determines an isomorphism of 2-

dimensional topological field theories between the Amodel of $M$ and the B-model of $\tilde{M}$, and vice versa. ${ }^{2}$

Readers familiar with topological field theory will know that the state space attached to the circle is a ring: these are the rings that appear in the quoted passage from [13].

Today, there is an ocean of literature on holomorphic maps from Riemann surfaces to Kähler, or more generally, symplectic manifolds, including Gromov-Witten invariants (the 'closed string' part of the story). The theory of Fukaya categories (the 'open string' part) is proceeding rapidly with respect to foundations and the development of tools. Laying down complete mathematical foundations

2 'Topological field theory should be understood in an 'extended' or 'open-closed' sense; $c f$. [5]. 
for the A-model topological field theory appears to be within reach. We also have a good formulation of the parts of the B-model where $\Sigma$ has genus 0 , incorporating derived categories and variations of Hodge structure, and an emerging understanding of the higher genus part $[5,14]$.

Counting curves. It seems that the germinal ideas of mirror symmetry elicited little more than skeptical shrugs from geometers. But in 1991, Candelas, de la Ossa, Greene, and Parkes [4] made a prediction which geometers could not ignore, for it seemed magical yet the evidence was compelling.

Taking the example of a quintic 3-fold $X \subset \mathbb{P}^{4}$, and a mirror consisting of a certain holomorphic 1-parameter family $\breve{X}_{q}$ of CY 3 -folds (the parameter $q$ varies in a punctured disc $\Delta^{*}=\{q \in \mathbb{C}: 0<|q|<1\}$ ) they studied a facet of SCFT visible in the topologically twisted A- and B-models and expected to match under mirror symmetry: the 3-point Yukawa couplings. For the A-model of $X$, the Yukawa coupling was identified as a generating function

$$
\mathrm{Y}_{A}(X)=5+\sum_{d=1}^{\infty} n_{d} d^{3} q^{d}\left(1+q^{d}+q^{2 d}+\ldots\right),
$$

where $n_{d}$ counts rational curves-the images of holomorphic maps $\mathbb{P}^{1} \rightarrow X$-meeting a hyperplane $H \subset \mathbb{P}^{4}$ with total multiplicity $d$. On the B-side, the Yukawa couplings are period integrals for the family $\left\{\check{X}_{q}\right\}$. Precisely, $\mathrm{Y}_{B}\left(\check{X}_{)}\right.$ is the Laurent series expansion of the holomorphic function on $\Delta^{*}$

$$
q \mapsto \int_{\check{X}_{q}} \check{\Omega} \wedge\left(q \frac{d}{d q}\right)^{3} \check{\Omega},
$$

where $\check{\Omega}$ is a holomorphic 3-form on the total space of the family, defining a volume form $\check{\Omega}_{q}$ on each fiber $\check{X}_{q}$; it has to be correctly normalized as a function of $q$. Candelas $e t$ al. computed that

$$
\mathrm{Y}_{B}(\check{X})=\frac{5}{\left(1+5^{5} x\right)} \frac{1}{y(x)^{2}}\left(\frac{q}{x} \frac{d x}{d q}\right)^{3},
$$

where

$y(x)=\sum_{n \geq 0} \frac{(5 n) !}{(n !)^{5}}(-1)^{n} x^{n}, \quad x(q)=-q+770 q^{2}+\ldots$.

The crucial change of coordinates $x=x(q)$, which they computed to all orders, is called the mirror map. Their prediction, then, was that

$$
\mathrm{Y}_{A}(X)=\mathrm{Y}_{B}(\check{X}) \text {. }
$$

They wrote:

It is gratifying that [assuming (2)] we find that $n_{1}=$ 2875 which is indeed the number of lines (rational curves of degree one) and $n_{2}=609250$ which is known to be the number of conics (rational curves of degree 2).
Mathematicians soon proposed a precise definition for the coefficients $N_{d}$ of the series

$$
\begin{aligned}
& \mathrm{Y}_{A}(X)=\sum N_{d} q^{d} \\
& \quad\left(\text { so } N_{0}=5, N_{1}=n_{1}, N_{2}=8 n_{2}+n_{1}, \text { etc. }\right) .
\end{aligned}
$$

It is rooted in Gromov's notion of pseudo-holomorphic curves in symplectic manifolds. One defines $N_{d}$ as a genuszero Gromov-Witten invariant, a homological 'count' of holomorphic maps $u: \mathbb{P}^{1} \rightarrow X$ of degree $d$, mapping three specified points $z_{j} \in \mathbb{P}^{1}(j=0,1,2)$ to $H_{j} \cap X$, where $H_{j} \subset \mathbb{P}^{4}$ is a specified hyperplane (Figure 2 ).
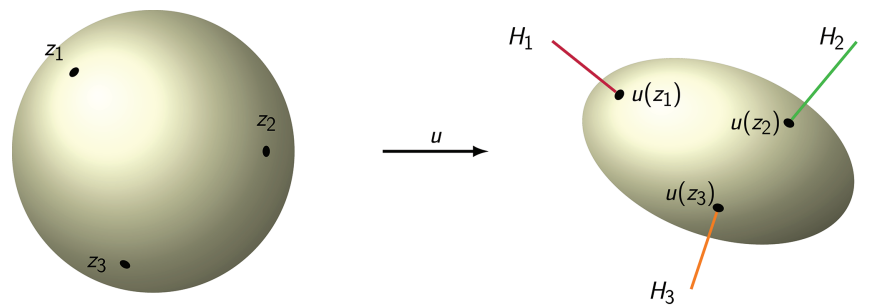

Figure 2. The A-side 3-point Yukawa coupling is a GW invariant enumerating holomorphic maps $u$.

GW invariants do not ultimately depend on the complex structure used on $X$ used to define them, so any smooth quintic 3-fold will serve. Such maps $u$ may factor through branched coverings $\mathbb{P}^{1} \rightarrow \mathbb{P}^{1}$, and there is a qualified sense in which the $n_{d}$ in (1) count the images, in $X$, of the maps $u$.

Principles. The intense activity inspired by the work of Candelas et al. made certain principles clear:

- The A-model of $(X, \omega, \Omega)$ concerns the symplectic geometry of $(X, \omega)$.

Gromov-Witten invariants-signed, weighted counts of holomorphic maps from Riemann surfaces into $X$ invoke a complex structure on $T X$, but this should be viewed as an auxiliary choice not affecting the outcome.

- The mirror to a CY manifold is not a single $\mathrm{CY}$ manifold, but a family of CY manifolds. The Bmodel concerns the complex analytic geometry of this family. ${ }^{3}$

The next principle is that one cannot expect mirror symmetry to arise from a single CY manifold $X$, nor from an arbitrary family. Rather,

- $X$ has a mirror when it undergoes a maximal degeneration to a singular variety, such as the degeneration of an elliptic curve to three projective lines (a degenerate cubic, Figure 3 ). ${ }^{4}$

\footnotetext{
${ }^{3}$ And, when these CY manifolds are projective varieties, their complex analytic geometry is interpretable as algebraic geometry.

${ }^{4}$ A maximal degeneration, parametrized by a small disc in $\mathbb{C}$, is one with maximally unipotent monodromy.
} 


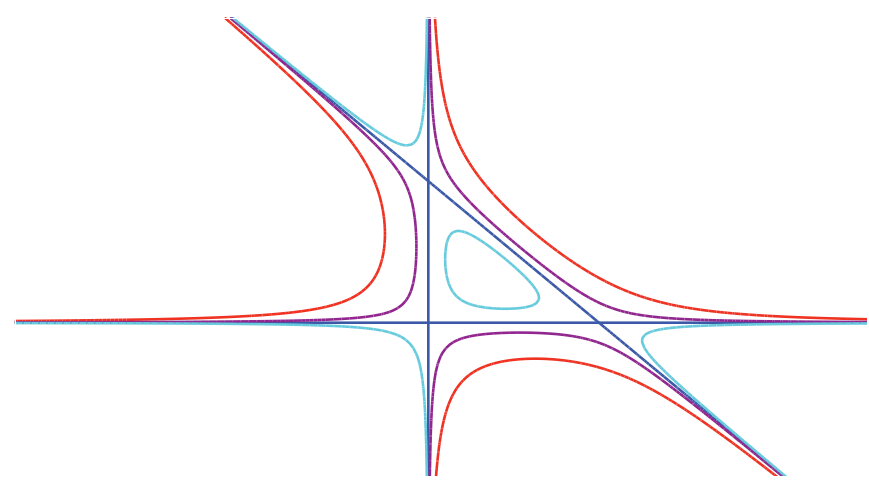

Figure 3. Plane cubic curves $x y(x+y-1)=\epsilon$ degenerating to three lines as $\epsilon \rightarrow 0$.

Finally, there is Kontsevich's eagle-eyed conjecture from 1994 [11], today called homological mirror symmetry (HMS), connecting Lagrangian submanifolds of $X$ to coherent sheaves on $\check{X}$. There are 'open string' topological field theories, governed by categorical structures called $A_{\infty}$ categories. In the A-model, one has the Fukaya $A_{\infty}$-category $\mathcal{F}(X, \omega)$ of the symplectic manifold $(X, \omega)$-its objects are Lagrangian submanifolds of $X$-and in the B-model, the bounded derived category $D(\check{X})$, whose objects are those complexes of sheaves $\check{\varphi}^{\bullet}$ of $\mathcal{O}_{\check{X}}$-modules whose cohomology sheaves $\mathcal{H}^{k}(\mathscr{\complement})$ are coherent and of bounded degree $k .^{5}$

We pause to define two of the terms:

Lagrangian submanifolds: A subspace $\Lambda$ of a vector space $V$ with a symplectic pairing $\omega_{V}$ is called Lagrangian if, for each $v \in \Lambda$, the linear form $\omega_{V}\left(\nu,{ }^{*}\right)$ vanishes precisely on $\Lambda$; this implies $\operatorname{dim} V=2 \operatorname{dim} \Lambda$. A submanifold $L \subset$ $M$ in a symplectic manifold $(M, \omega)$ is one whose tangent spaces $T_{X} L$ are Lagrangian in $T_{X} M$.

Coherence of sheaves: In algebraic geometry, and similarly in the rigid analytic geometry we shall discuss later, an algebraic variety $Z$ comes with a sheaf of rings $\mathcal{O}_{Z}$, the structure sheaf, assigning a commutative ring $\mathcal{O}_{Z}(U)$ to each open set $U \subset Z$. A sheaf of $\mathcal{O}_{Z}$-modules $\mathscr{\wp}$ assigns an $\mathcal{O}_{Z}(U)$-module $\mathscr{\complement}(U)$ to each open $U$. Assuming for simplicity that $\mathcal{O}_{Z}(U)$ is a Noetherian ring for small neighborhoods $U$ of an arbitrary point $z \in Z$, we say $\mathscr{E}$ is coherent if each point of $Z$ has an open neighborhood $U$ such that (i) the $\mathcal{O}_{Z}(U)$-module $\mathscr{\complement}(U)$ is finitely generated; and (ii), for all open sets $V \subset U$, the map $\mathcal{O}_{Z}(V) \otimes_{\mathcal{O}_{Z}(U)} \mathscr{E}(U) \rightarrow$ $\mathscr{\wp}(V),\left.f \otimes s \mapsto f \cdot s\right|_{V}$, is an isomorphism.

- HMS: There is a functor $\mathcal{F}(X, \omega) \rightarrow D(\check{X})-$ mapping Lagrangian submanifolds of $X$ to coherent complexes of sheaves on $\check{X}$-which is, in a certain sense, a categorical equivalence. ${ }^{6}$

\footnotetext{
${ }^{5}$ The derived category should here be treated not as a triangulated category, but its enhancement to a differential-graded (hence $A_{\infty}$ ) category.

${ }^{6}$ Namely, it induces a quasi-equivalence of the associated $A_{\infty}$-categories of right modules. It may appear that HMS is incompatible with the notion that the mirror is a family. When HMS is formulated more precisely, this apparent disconnect proves illusory.
}

Kontsevich foresaw that HMS should be an organizing principle; that it should imply the isomorphism of topological field theories $A(X)$ and $B(\check{X})$, and thereby enumerative statements such as the prediction (2).

Verification, explanation. Some of mirror symmetry's predictions were soon verified. Candidate mirror partners were found for many CY manifolds. The Yukawa couplings $\mathrm{Y}_{A}(X)$ were computed for a class of CY manifolds $X$ including the quintic 3-fold [7] by showing that they satisfy the same differential equations as their B-side counterparts $\mathrm{Y}_{B}(\check{X})$. Such work bore witness to the mirror symmetry phenomenon, but did not explain it.

Explanations gradually emerged $[5,12,17]$. The GrossSiebert program [10] is a systematic and sophisticated construction of mirror pairs, for which several of the predictions of mirror symmetry have been proven. HMS has recently become tractable as basic tools for working with Fukaya categories have been developed. We now know [8] that HMS is an indeed an organizing principle, implying statements such as (essentially) (2). We know that HMS is true for (on the A-side) the quintic 3 -fold [16], and we have a prototype for a truly explanatory proof of HMS $[1,2]$.

\section{The Key Questions}

(a) How do we construct a mirror $\check{X}$ to a CY manifold $X$ ?

(b) How can the symplectic geometry of $X$ be read as analytic geometry of $\check{X}$-or vice versa?

(c) Why is HMS true?

(d) Why is mirror symmetry involutory?-Why is $X$ the mirror of its mirror $\check{X}$ ?

The germ of the answer to (a) and (d) was proposed by Strominger-Yau-Zaslow (SYZ) in 1996 [17]. The point is to find a smooth, surjective map

$$
f: X^{2 n} \rightarrow Q^{n}
$$

to a middle-dimensional base $Q$ such that the subspace ker $D_{x} f \subset T_{X} X$ is Lagrangian for all regular points $X$; so the regular fibers are Lagrangian submanifolds of $X$.

The regular fibers $F_{q}:=f^{-1}(q)$ are necessarily tori: each fiber $F_{q}$ has the structure of an $n$-dimensional affine vector space $A$ modulo the action of a lattice $L$ in its vector space $V$ of translations. One then obtains the mirror $\check{X}$ by replacing the non-singular fibers of that family by the dual tori $\breve{F}_{q}:=H^{1}\left(F_{q} ; \mathbb{R} / \mathbb{Z}\right) \cong V^{*} / L^{*}$, the quotients of the dual vector spaces by the dual lattices. Provided one can find a way to handle the singular fibers, one obtains in this way a space $\breve{X}$ and a map $\breve{f}: \breve{X} \rightarrow Q$ with fibers $\breve{F}_{q}$.

A CY manifold $X$ admits an 'optimal' pair $(\omega, \Omega)$, one for which $\Omega$ is covariantly constant with respect to the Kähler metric: this is a famous theorem of S.-T. Yau. A Lagrangian $L \subset X$ is called special with respect to a CY 


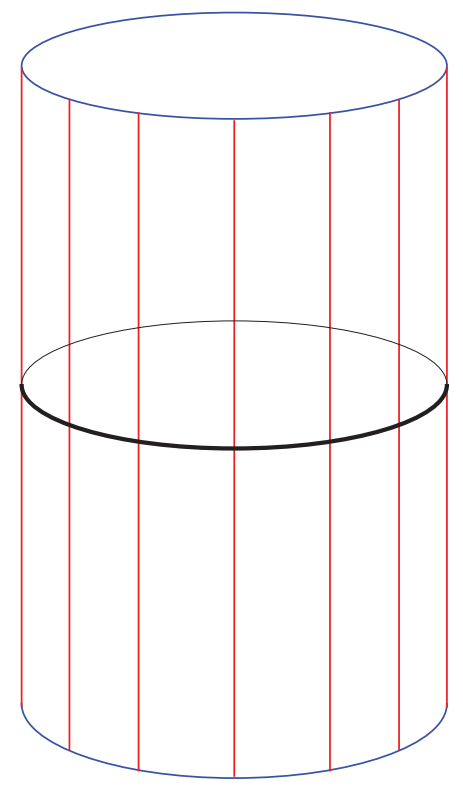

Figure 4. A schematic of $T^{*} S^{1}$; the top and bottom are glued together to form $\left(T^{*} S^{1}\right) /\left(T^{*} S^{1}\right)_{\mathbb{Z}}$. The red lines are the cotangent fibers, which become circles in the quotient.

metric if the imaginary part of $\Omega$ vanishes on $\Lambda^{n} T L$. SYZ proposed that the fibers $F_{q}$ should be special Lagrangian. Orientable special Lagrangians admit phase functions $\phi$ : there is a non-vanishing section $v_{L}$ of $\Lambda^{n} T L$ such that $\arg \Omega\left(\nu_{L}\right): X \rightarrow S^{1}$ admits a continuous logarithm $\phi$, called a phase function. Lagrangians with phase functions will suffice for our needs in this article, and special Lagrangians will not reappear.

The basic model for the SYZ mirror-disregarding specialness-is as follows:

Let $Q$ be an integral affine $n$-manifold, that is, an $n$-manifold with an atlas of charts whose transition functions are affine transformations between open subsets of $\mathbb{R}^{n}$, of shape $x \mapsto A x+b$ with $b \in \mathbb{R}^{n}$ and $A \in G L_{n}(\mathbb{Z})$. The cotangent bundle $T^{*} Q$ is naturally a symplectic manifold, and there is a natural integer lattice $\left(T_{q}^{*} Q\right)_{\mathbb{Z}}$ in each cotangent space $T_{q}^{*} Q$. Let $X=\left(T^{*} Q\right) /\left(T_{\mathbb{Z}}^{*} Q\right)$ be the quotient of $T^{*} Q$ by fiberwise-translations by lattice-vectors (Figure 4). Then $X$ is symplectic, and is a bundle of Lagrangian $n$-tori over $Q$. The tangent bundle $T Q$ contains a lattice dual to the one in $T^{*} Q$, and the quotient manifold $\check{X}=T Q / T_{\mathbb{Z}} Q$ is naturally complex. Then $X$ and $\check{X}$ are mirrors.

SYZ's idea is at the heart of our current understanding of mirror symmetry, but the version I will outline in the section on rigid analytic mirrors is purely symplectic rather than Riemannian in nature, and, unlike the basic model just presented, it makes $\breve{X}$ a complex 1-parameter family.

\section{Prototypes: Fourier Transforms, Classical and Geometric}

Pontryagin duality. The most basic model for a duality such as mirror symmetry is the passage from a finitedimensional vector space to its dual. A more instructive example is Pontryagin duality. The characters of a locally compact, abelian topological group $G$ are the continuous homomorphisms $G \rightarrow \mathbb{T}$ to the circle-group $\mathbb{T}=\mathbb{R} / \mathbb{Z}$. In Fourier analysis, one takes $G=\mathbb{R}$ or $\mathbb{T}$, so that the respective characters are the maps $X \mapsto e^{2 \pi i t x}$ for $t \in \mathbb{R}$ or $t \in \mathbb{Z}$. The set $\hat{G}$ of characters is again a locally compact topological group, the Pontryagin dual of $G$. There is a 'universal character', which is the evaluation pairing $X: \hat{G} \times$ $G \rightarrow \mathbb{T}, \chi(\xi, x)=\xi(x)$. A complex-valued function $f$ on $G$ has a Fourier transform $\hat{f}$, a function on $\hat{G}: \hat{f}(\xi)=$ $\int_{G} \overline{\chi(\xi, x)} f(x) \mu_{G}$, where $\mu_{G}$ is a suitably-normalized leftinvariant measure defined on the open sets.

The construction is a duality inasmuch as the evaluation map ev : $\hat{\hat{G}} \rightarrow G$ is an isomorphism, and $\hat{\hat{f}}=f \circ$ inv $\circ$ ev (where inv: $G \rightarrow G$ is inversion).

Mirror symmetry, based on the SYZ idea, is roughly analogous to the formation of the Pontryagin dual group, with the Fourier transform a prototype for HMS.

Fourier-Mukai transforms for K3 surfaces. Fourier-Mukai transforms [15] bring us closer to mirror symmetry proper. Consider a simply connected, compact CY complex surface $(S, \omega, \Omega)$ embedded in a projective space: a projective $K 3$ surface.

Holomorphic vector bundles, or more generally, coherent sheaves $\mathcal{F}$, over $S$, have a discrete invariant, the Chern character, which is best packaged as the Mukai vector $\nu(\mathcal{F})=v_{0}+v_{2}+v_{4} \in H^{0}(S ; \mathbb{Z}) \oplus H^{2}(S ; \mathbb{Z}) \oplus H^{4}(S ; \mathbb{Z}) .^{7}$ There is a moduli space $M_{S, v}$, parametrizing isomorphism classes of 'stable' coherent sheaves $\mathcal{F}$, with fixed Mukai vector $v$; under assumptions that go unstated here, it is a compact complex manifold, projective, of dimension $2+$ $(v, v)$, where $(v, v)=\int_{S}\left(-2 v_{0} v_{4}+v_{2}^{2}\right)$. In the isotropic case $(v, v)=0, M_{S, v}$ is again a surface, and is again CY. ${ }^{8}$ In the case that $v=1 \in H^{0}(S ; \mathbb{Z})$, one has $M_{S, v}=S$, the points of $M_{S, v}$ being merely the ideal sheaves for the points $S \in S$. But for other choices of Mukai vector, $M_{S, v}$ is a new $\mathrm{K} 3$ surface, and we can recover $S$ as a moduli space of sheaves of $M_{S, v}$ :

$$
M_{S, v} \cong M_{M_{(S, v)}, v^{\prime}}
$$

for a certain Mukai vector $v^{\prime}$ for $M_{S, v}$. Thus a moduli space of geometric objects on a $\mathrm{K} 3$ surface gives rise to a new $\mathrm{K} 3$ surface, in a reciprocal relationship with the original.

\footnotetext{
${ }^{7}$ The Mukai vector is $\mathrm{ch}(\mathcal{F}) \wedge(1+\eta)$, where $\mathrm{ch}$ is the Chern character and $\eta$ is the generator for $H^{4}(S ; \mathbb{Z})$.

${ }^{8}$ The holomorphic volume form is the Serre duality pairing on $T_{\mathcal{F}} M_{S, v}=\operatorname{Ext}_{\mathcal{O}_{S}}^{1}(\mathcal{F}, \mathcal{F})$
} 
There is a distinguished sheaf on $S \times M_{S, v}$, the universal sheaf $\mathcal{E}_{\text {univ, }}$ whose restriction to the slice $S \times\{\mathcal{F}\}=S$ is isomorphic to $\mathcal{F}$. ${ }^{9}$ The Fourier-Mukai transform now inputs coherent sheaves $\mathcal{E}$ on $S$, and outputs (complexes of) coherent sheaves on $M_{S, v}$ :

$$
\mathcal{F} \mapsto \hat{\mathcal{F}}=\left(\mathrm{pr}_{2}\right)_{*}\left(\mathcal{E}_{\text {univ }} \otimes \mathrm{pr}_{1}^{*} \mathcal{F}\right) .
$$

The Fourier-Mukai transform has a categorical manifestation, which is strongest when $(v, v)=0$ : it then defines an equivalence of derived categories of coherent sheaves on $S$ and on $M_{S, v}$. This is the model for HMS.

\section{Rigid Analytic Mirrors}

The Novikov field and rigid analytic geometry. Fix a field $F$. The vector space $F^{\mathbb{R}}$ of all functions $\lambda: \mathbb{R} \rightarrow F$ has a subspace $\Lambda_{F}$ of Novikov series: functions $\lambda$ whose support is discrete and bounded below. One can multiply Novikov series, by convolution; thus we usually write Novikov series as formal series

$$
\begin{aligned}
\lambda=\sum_{j=1}^{\infty} \lambda_{j} q^{r_{j}}, & \lambda_{j} \in F, \\
& r_{j} \in \mathbb{R}, \quad r_{1}<r_{2}<\ldots, \quad r_{j} \rightarrow \infty .
\end{aligned}
$$

(This series represents the function supported on $\left\{r_{1}\right.$, $\left.r_{2}, \ldots\right\}$ given by $r_{j} \mapsto a_{j}$.) In this way $\Lambda_{F}$ becomes a field; the complex Novikov field $\Lambda_{\mathbb{C}}$ is algebraically closed.

The most important feature of $\Lambda_{F}$ is that it comes with a complete valuation

$$
\operatorname{val}(\lambda):=\min \operatorname{supp} \lambda \text {. }
$$

A valuation on a field $K$ is a map val : $K^{\times} \rightarrow \mathbb{R}$ (extended to $K$ by setting val $(0)=+\infty)$ such that $\operatorname{val}(x+y) \geq$ $\min (\operatorname{val}(x), \operatorname{val}(y))$ and $\operatorname{val}(x y)=\operatorname{val}(x)+\operatorname{val}(y)$. There is an associated absolute value, $|x|=\exp (-\operatorname{val}(x))$, and a metric $d(x, y)=|x-y|$. The valuation is complete if $d$-Cauchy sequences converge.

Rigid analytic geometry [18] is a variant of algebraic geometry, applicable over a complete valued field ( $K$, val): it builds in the internal geometry of the valuation.

In algebraic geometry over a field $K$-which, for brevity, we here assume algebraically closed-the basic objects are polynomial algebras $K\left[Z_{1}, \ldots, Z_{n}\right]$. Maximal ideals therein correspond to points $x \in K^{n}$, as they take the form ( $z_{1}-$ $\left.x_{1}, \ldots, z_{n}-x_{n}\right)$. In rigid analytic geometry, one instead studies the Tate algebra $T_{n}=K\left\langle Z_{1}, \ldots, Z_{n}\right\rangle$, the algebra of power series $f(z)=\sum f_{I} z^{I}$, a sum over multi-indices $\left(i_{1}, \ldots, i_{n}\right) \in\left(\mathbb{Z}_{\geq 0}\right)^{n}$, with $f_{I} \in K$ and $z^{I}=\prod z_{j}^{i_{j}}$, such that $\left|f_{I}\right| \rightarrow 0$ as $\|I\| \rightarrow \infty$, where $\|I\|=\sum_{j} i_{j}$. If one has a point $x=\left(x_{1}, \ldots, x_{n}\right)$ in the 'unit polydisk' $\mathbb{D}^{n} \subset K^{n}$, meaning $\left|x_{j}\right| \leq 1$ for all $j$, it defines a maximal ideal $\mathfrak{m}_{x}=\left(z_{1}-x_{1}, \ldots, z_{n}-x_{n}\right) \subset T_{n}$ : there is

${ }^{9}$ Mukai develops 'quasi-universal sheaves' in cases where automorphisms preclude a universal sheaf. an isomorphism $T_{n} / \mathfrak{m}_{x} \rightarrow K$, given by $[f] \mapsto f(x)=$ $\sum_{j \geq 0} \sum_{\|I\|=j} f_{I} x^{I}$ (convergent series). This construction accounts for all maximal ideals of $T_{n}$, and so one thinks of $T_{n}$ geometrically as the polydisk $\mathbb{D}^{n}$.

A quotient $A=K\left[z_{1}, \ldots, z_{n}\right] /\left(f_{1}, \ldots, f_{m}\right)$ determines a topological space $X=\operatorname{Spec} A$. The points of $X$ are the prime ideals of $A ; X$ has its Zariski topology, in which the maximal ideals are the closed points. One thinks of the closed points of $X$ as the zero-set $f_{1}(x)=\cdots=f_{m}(x)=0$ inside $K^{n}$. There is a $K$-algebra of 'functions' $\mathcal{O}_{X}$ on $X$, the maps $x \mapsto a(x) \in A / \mathfrak{m}_{x}$ where $a \in A$ and $x \in X$ labels a maximal ideal $\mathfrak{m}_{x}$. But actually, $\mathcal{O}_{X} \cong A$.

Likewise, a quotient $A=T_{n} /\left(f_{1}, \ldots, f_{m}\right)$ determines a space $X=\mathrm{Sp} A$ of maximal ideals, called an affinoid space. As before, it determines $A$ as its ring of functions $\mathcal{O}_{X}$.

Certain subsets $U \subset X$ inside an affinoid space $X=$ $\mathrm{Sp} A$ are called affinoid domains. Take a (suitable) norm $\|\cdot\|$ on $A$, and the induced norms $\|\cdot\|_{X}$ on its quotients $A / \mathfrak{m}_{X}:\|a\|_{X}=\inf \left\{\|b\|: b-a \in \mathfrak{m}_{x}\right\}$. Then, for $f \in A$ and $c \in \mathbb{R}$, the set $X(f, c)=\left\{x \in X:\|f(x)\|_{x} \leq\right.$ $c\}$ is an affinoid domain. So too is a finite intersection $\bigcap X\left(f_{j}, c_{j}\right)$.

In algebraic geometry, spectra of $K$-algebras can be 'glued' together to form a global object, a $K$-scheme, which is a topological space $Z$ equipped with a sheaf $\mathcal{O}_{Z}$ of $K$ algebras, locally the spectrum of a $K$-algebra. Tate showed how affinoid subdomains of affinoid spaces can be glued together to form a global object-a space $Z$ with a sheaf of $K$-algebras $\mathcal{O}_{Z}$, which is locally the algebra of functions of an affinoid domain.

Rigid analytic mirrors. Suppose we have a compact, convex polytope $P \subset \mathbb{R}^{n}$. To this we attach the set

$$
\check{X}_{P}=\left\{x \in\left(\Lambda_{\mathbb{C}}^{\times}\right)^{n}:\left(\operatorname{val}\left(x_{1}\right), \ldots, \operatorname{val}\left(x_{n}\right)\right) \in P\right\}
$$

(Figure 5). This subset is actually an affinoid subdomain of an affinoid space over the Novikov field $\Lambda_{\mathbb{C}}$. First, we can realize the annular domain $\left\{x \in \Lambda_{\mathbb{C}}^{n}: \epsilon \leq\left|x_{j}\right| \leq\right.$ $\left.\epsilon^{-1}, j=1, \ldots, n\right\}$ as an affinoid space $A_{\epsilon}^{n}$. The polytope $P$ is cut out from $\mathbb{R}^{n}$ by a finite list of inequalities, each of shape $\lambda \cdot x \geq c$, where $\lambda \in \mathbb{Z}^{n}$ and $c \in \mathbb{R}$. And $\check{X}_{P}$ is cut out, inside $A_{\epsilon}^{n}$ for a suitably small $\epsilon$, by inequalities $\left|x_{1}^{\lambda_{1}} \cdots x_{n}^{\lambda_{n}}\right| \leq e^{-c}$; this identifies it as an affinoid subdomain of $A_{\epsilon}^{n}$.

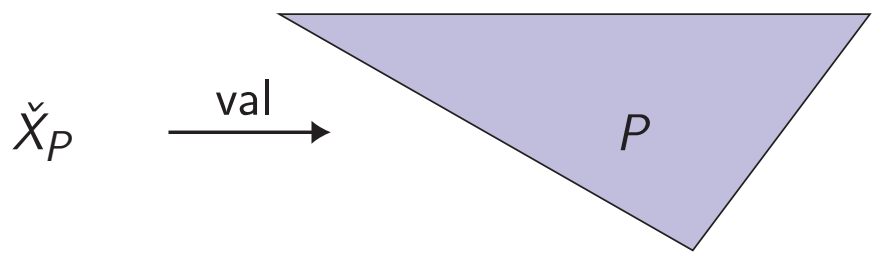

Figure 5. The values of the coordinates of the affinoid domain $\check{X}_{P}$ form the polytope $P$. 
Suppose now that one has an $n$-manifold $Q$ which is not merely smooth, but integral affine (cf. 'The Key Questions') - such as the base of a fibering of a symplectic manifold $X$ by Lagrangian submanifolds $\left\{F_{q}\right\}_{q \in Q}$. "Triangulate $Q$ by a collection of integral affine polytopes $P_{\alpha}$. Each of them defines an affinoid domain $\check{X}_{P_{\alpha}}$, and these glue together to form a rigid analytic space $\check{X}_{\text {over }} \Lambda_{\mathbb{C}}$, which does not change when one subdivides the triangulation.

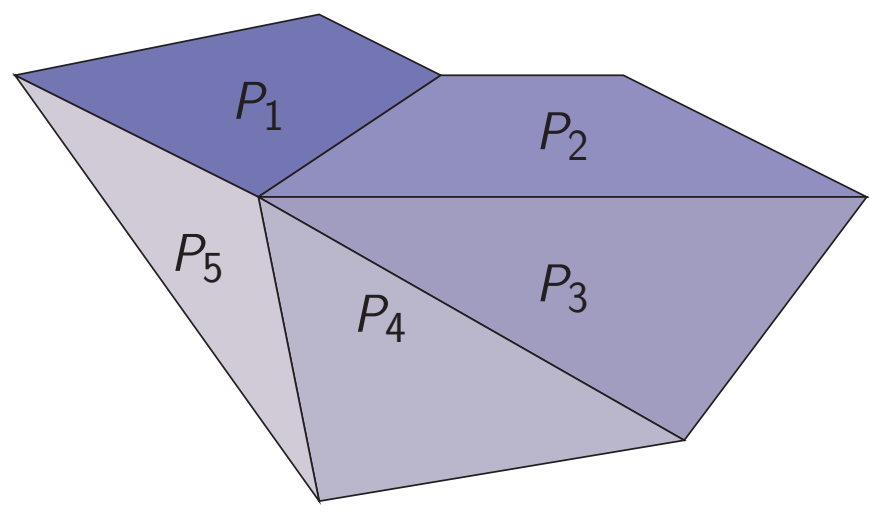

Figure 6. Fragment of a triangulation of $Q$, as it appears in an integral affine chart.

The set underlying $\check{X}$ is the space of pairs $(q, \eta)$, where $q \in Q$ and $\eta \in H^{1}\left(F_{q} ; U(\Lambda)\right)$. Here $U(\Lambda)=$ $\left\{\lambda \in \Lambda^{\times}:|\lambda|=1\right\}$ : so the mirror is a space of pairs of a torus-fiber $F_{q}$ and a homomorphism from the first homology group $H_{1}\left(F_{q}\right) \cong \mathbb{Z}^{n}$ to the group of unit-norm Novikov series-made into a rigid analytic space.

For example, if $Q=\mathbb{R} / \mathbb{Z}$ is the circle-the base of a Lagrangian fibration on the 2-torus $\mathbb{R}^{2} / \mathbb{Z}^{2}$ viewed as a symplectic manifold-its affine integral structure is inherited from $\mathbb{R}$, and we can triangulate it by intervals $[a, b]$. The affinoid domain associated with an interval is an 'annulus' $\left\{z \in \Lambda^{\times}: e^{-b} \leq|z| \leq e^{-a}\right\}$, and these glue together to form an elliptic curve over $\Lambda$, the Tate curve $E_{\text {Tate }}=\Lambda^{\times} / q^{\mathbb{Z}}$.

Pseudo-holomorphic curves. Why should rigid analytic geometry over the Novikov field have anything whatsoever to do with symplectic topology? The brief answer is: Gromov compactness.

Symplectic topologists probe symplectic manifolds $(X, \omega)$ using pseudo-holomorphic curves: maps $u: \Sigma \rightarrow X$ from a Riemann surface $\Sigma$ to $X$ such that, for some specified complex structure $J$ on $T X$, the derivative $D u$ is complex linear. Thus, if $j$ is the complex structure on $T \Sigma$, one has the 'Cauchy-Riemann equation' $J \circ D u=D u \circ j$. In the presence of a Lagrangian submanifold $L \subset X$, one may suppose that $\Sigma$ has boundary, and impose the boundary condition that $\partial u$ (the restriction of $u$ to the boundary $\partial \Sigma$ ) maps $\partial \Sigma$ to $L$.

Once one pins down the smooth surface underlying $\Sigma$, and the Lagrangian boundary conditions, there is a moduli space $\mathcal{M}$ of pseudo-holomorphic curves in $X$, which one should think of as a smooth manifold. One can also allow pseudo-holomorphic curves with nodal domains, and from these one can construct a larger moduli space $\overline{\mathcal{M}}$. Gromov compactness says that the subspace $\overline{\mathcal{M}}_{\leq c}$, where the energy $E(u)=\int_{\Sigma} u^{*} \omega$ is at most $c$, is compact.

One typically imposes conditions on $u$ so as to cut $\overline{\mathcal{M}}$ down to a zero-dimensional manifold $N$. Then the compact sub-level sets $N_{\leq c}$ for the energy function $E$ are finite. Once one has a recipe for orienting $N$, one can 'count' its points with signs, and the result is a Novikov series, $\# N:=\sum_{u \in N} \operatorname{sign}(u) q^{E(u)} \in \Lambda_{\mathbb{C}}$.

From Lagrangians to coherent sheaves. Suppose that we have a compact CY manifold $\left(X^{2 n}, \omega, \Omega\right)$ and a nonsingular fibering $f: X^{2 n} \rightarrow Q^{n}$ by Lagrangian submanifolds-necessarily tori-which admit phase functions. Then $Q$ acquires an integral affine structure. Suppose also that we have identified a section $\sigma: Q \rightarrow X$ of $f$ whose image is Lagrangian; then $X=T^{*} Q /\left(T^{*} Q\right)_{\mathbb{Z}}$. As we discussed in the section on rigid analytic mirrors, we can use the integral affine structure of $Q$ to define a rigid analytic $\Lambda$-space $\check{X}=\check{X}_{\text {rigid }}$. This is our mirror. ${ }^{10}$ It comes with a natural map $\breve{f}: \check{X} \rightarrow Q$, and the fiber $\breve{f}^{-1}(q)$ can be identified with $H^{1}\left(F_{q} ; U_{\Lambda}\right)$, where $U_{\Lambda}=$ val $^{-1}(0) \subset \Lambda^{\times}$ is the group of unit-norm Novikov series.

Now we come to the 'Fourier transform' underlying HMS, the process by which Lagrangians are converted into coherent sheaves on the mirror. Suppose $L \subset X$ is a compact Lagrangian submanifold, equipped with a phase function. One then defines sheaves $\mathcal{H}^{k}\left(\mathscr{\complement}_{L}\right)$ of $\mathcal{O}_{\check{X}}$-modules on $\check{X}$ : Cover $Q$ by integral polytopes $P_{\alpha}$, and let $q_{\alpha} \in P_{\alpha}$ be a reference point. For each $\alpha$, we can perturb $L$ to a new Lagrangian $L_{\alpha}$ such that $L_{\alpha} \cap F_{q}$ is a transverse intersection for every $q \in P_{\alpha}$. We define a module $\mathscr{\wp}_{L, \alpha}$ over the ring of functions $\mathcal{O}_{\alpha}:=\mathcal{O}_{\check{X}_{P_{\alpha}}}$ of $\check{X}_{P_{\alpha}}$ by

$$
\mathscr{\wp}_{L, \alpha}=\left(\mathcal{O}_{\alpha}\right)^{L_{\alpha} \cap F_{q_{\alpha}}}:
$$

the free module on the set of intersection points. The module $\mathscr{\wp}_{L, \alpha}$ has a grading, defined via phase functions, and a differential $\delta$-a square-zero endomorphism which increases the grading by 1 . The construction of $\delta$ uses family Floer cohomology. It involves pseudo-holomorphic bigons, $\operatorname{discs} \bar{\Delta} \rightarrow X$, with a boundary condition that requires the upper half of $\partial \bar{\Delta}$ to map to $L_{\alpha}$, and the lower half to $F_{q}$

\footnotetext{
${ }^{10}$ An important and delicate issue is whether there are holomorphic discs in $X$ whose boundary lies on a fiber of $f$, and if so, how properly to account for them in the construction of the mirror. For present purposes, assume there are none. This assumption is a major simplification of what is typically true.
} 
for some $q \in P_{\alpha}$. For present purposes, we assume an absence of holomorphic discs whose entire boundary lies on $F_{q}$ or $L_{\alpha}$. This is vital; to make things work in generality, one will need to prove their absence rather than assuming it. The fact that $\delta$ makes sense expresses a compatibility between pseudo-holomorphic curves and rigid analytic geometry $[2,6]$.

We then pass to the cohomology module

$$
H^{*}\left(\mathscr{\complement}_{L, \alpha}\right)=\operatorname{ker} \delta / \operatorname{im} \delta .
$$

This is a finitely generated $\mathcal{O}_{\alpha}$-module. While patterns of intersections change under perturbations of Lagrangians, $H^{*}\left(\mathscr{E}_{L, \alpha}\right)$ does not depend on the perturbation $L \leadsto L_{\alpha}$. One can use that fact to assemble the modules $H^{*}\left(\mathscr{\wp}_{L, \alpha}\right)$ into a sheaf $\mathcal{H}^{*}\left(\mathscr{\complement}_{L}\right)$ of $\mathcal{O}_{\check{X}}$-modules. Locally, it is the sheaf associated with a finitely generated module over a Noetherian ring-so it is coherent.

The mapping $L \mapsto \mathcal{H}^{*}\left(\mathscr{\wp}_{L}\right)$, sending a Lagrangian to a coherent sheaf on the rigid analytic mirror, is the 'Fourier transform' which explains HMS [2].

\section{Mirror Symmetry as an Operation on Holomor- phic Families}

We have just seen that the symplectic geometry of families of Lagrangian submanifolds, fibering $X$, gives rise to a rigid analytic mirror $\check{X}_{\text {rigid }}$ over the complex Novikov field $\Lambda$, and that other Lagrangians in $X$ then produce coherent analytic sheaves on $\breve{X}_{\text {rigid }}$. But a rigid analytic space is not a symplectic manifold, so this cannot be an involutory process like Pontryagin duality or the Fourier-Mukai transform.

I want to outline, via an example, how the formation of rigid analytic mirrors should feed into an involutory procedure, not yet fully understood, the construction of the mirror partner to a degenerating 1-parameter families of CY manifolds, whereby the mirror of the mirror is the original.

The first point is that degenerations should give rise to Lagrangian torus fibrations. Start with projective space $\mathbb{P}^{d}$. This has a Lagrangian torus fibration $\mathbb{P}^{d} \rightarrow \Sigma_{d}$, of sorts, whose fibers are 'Clifford tori,' the points $\left(z_{0}: \cdots: z_{d}\right)$ with $\sum\left|z_{k}\right|^{2}=1$ and $\left|z_{j}\right|=c_{j}$ (constant) for each $j$. The base $\Sigma_{d}$ is a $d$-dimensional simplex. Some of the Clifford tori, those lying over the boundary of the simplex, are not Lagrangian, because they are tori of dimension less than d.

Now consider the 'totally degenerate CY hypersurface' $X_{0}=\left\{z_{0} \cdots z_{d}=0\right\} \subset \mathbb{P}^{d+1}$. It is a union of $d+1$ projective hyperplanes $x_{k}=0$, and the Lagrangian torus fibrations over these hyperplanes assemble to give a map $\mu: X_{0} \rightarrow P$ to a $d$-dimensional polyhedron formed by gluing the $d+1$ simplices along faces $(P$ actually just the boundary of a $(d+1)$-dimensional simplex). The fibers of $\mu$ are Lagrangian tori over the interiors of the faces of $P$, and are lower-dimensional tori elsewhere (Figure 7).

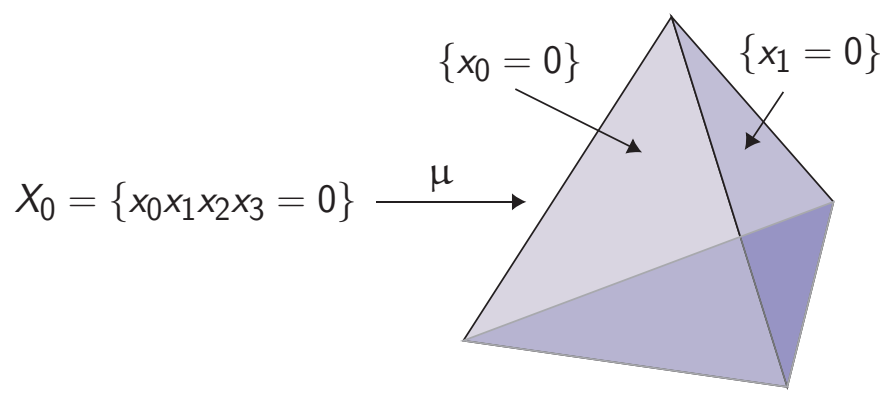

Figure 7. The map $\mu: X_{0} \rightarrow P$ in the case $d=2$.

Next, consider the family of CY hypersurfaces

$$
X_{t}=\left\{(t, z) \in \mathbb{C} \times \mathbb{P}^{d+1}: t F(z)+z_{0} \cdots z_{d}=0\right\},
$$

where $F$ is a (generic) homogeneous polynomial of degree $d+1$. Thus $X_{1}$ is a CY manifold, while $X_{0}$ is our singular, totally degenerate $\mathrm{CY}$ hypersurface. One can use the symplectic geometry of the family (with a Kähler form inherited from $\mathbb{C} \times \mathbb{P}^{d+1}$ ) to produce a map $\rho: X_{1} \rightarrow X_{0}$ which is a symplectomorphism over the smooth locus in $X_{0}$. The composite $f: X_{1} \stackrel{\rho}{\rightarrow} X_{0} \stackrel{\mu}{\rightarrow} P$ is then our candidate for a Lagrangian torus fibration. Over the interiors of the simplices of $P, \mu$ has Lagrangian fibers and $\rho$ is a diffeomorphism; over a codimension $k$ facet of $P$, the fibers of $\mu$ have dimension $d-k$, but those of $\rho$ have dimension $k$, so $f$ has fibers of dimension $d$, as we want. However, there is a 'bad' locus $B \subset X_{0}$ where the total space of the family is singular, and the mechanism breaks down; that is the source of singularities in the fibers of $f$ (Figure 8).

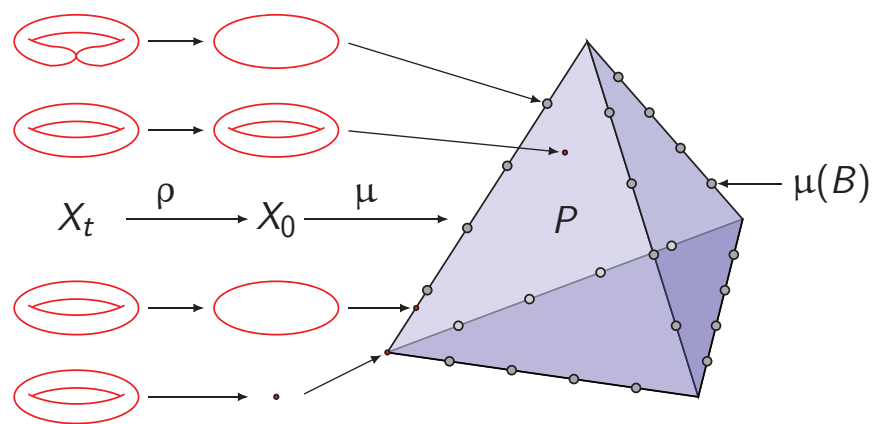

Figure 8. The map $f: X_{t} \rightarrow P$ in the case $d=2$, showing some of its fibers in red. The 24 dots on the edges of the tetrahedron $P$ are the images of the singular locus of the total space of the family. 
This example illustrates a mechanism whereby toric degenerations of CY manifolds-roughly, degenerations to varieties each of whose irreducible components is a toric variety-should give rise to Lagrangian torus fibrations. ${ }^{11}$

The fiber $X_{1}$ comes with a symplectic automorphism $m$, the monodromy around the unit circle, which-in a model situation, at any rate-preserves the fibers of $f$, and acts as translation of each of the non-singular fibers. This automorphism corresponds to extra structure on the mirror, a line bundle over $\breve{X}_{\text {rigid }}$. One expects that this line bundle is ample, and therefore defines an embedding of $\breve{X}_{\text {rigid }}$ into rigid analytic projective space. Just as in complex analytic geometry, the image of an embedding into projective space is in fact cut out algebraically by polynomials-so the $\check{X}_{\text {rigid }}$ becomes an algebraic scheme $\check{X}_{\text {alg }}$ over $\Lambda_{\mathbb{C}}$.

Pause for a moment to observe that if we have a family $Z_{t}$ of complex projective varieties, whose defining equations depend holomorphically on $t \in \Delta^{*}$ (the punctured disc), we can take the Laurent expansions of these equations to get a family $z$ over the field $\mathbb{C}((t))$ of finite-tailed Laurent series, and therefore, by extending scalars, a variety over $\Lambda_{\mathbb{C}}$. One can ask whether $\check{X}_{a l g}$ arises in this way, from a family $\check{X}_{t}$ of complex projective varieties. This is not the place to get into the details, but there are geometric reasons to expect that to be true. In this way, we end up with a new family $\left\{\breve{X}_{t}\right\}$ of complex projective CY manifolds, mirror to the original family.

While the general picture described here has large gaps still to be filled, an algebro-geometric analogue of the composite process has been fully worked out by Gross-Siebert [10]. Their works centers on a part of the story called wallcrossing that I have not even hinted at.

Example. If one takes a degenerating family of elliptic curves $X \rightarrow \Delta^{*}$, given as cubic curves in $\mathbb{P}^{2}$, the generic fiber $X$ is (symplectically) the 2-torus $\mathbb{R}^{2} / \mathbb{Z}^{2}$ and it has the Lagrangian fibration given by projection $f: \mathbb{R}^{2} / \mathbb{Z}^{2} \rightarrow$ $\mathbb{R} / \mathbb{Z}$. After choosing a section of $f$, one obtains the Tate curve as rigid analytic mirror, with a degree 1 line bundle over it. Section of powers of this line bundle define an embedding of the Tate curve into $\mathbb{P}^{2}(\Lambda)$ as a cubic curve

$$
y^{2}+x y=x^{3}+a_{4}(q) x+a_{6}(q),
$$

where $a_{4}$ and $a_{6}$ are certain power series in $q$. In particular, this curve is defined over $\mathbb{C}((q))$. Since $a_{4}$ and $a_{6}$ are convergent in the unit disc $|q|<1$, it can also be viewed as a holomorphic family over $\Delta^{*}$-the mirror to the original family.

\footnotetext{
${ }^{11}$ This mechanism was first explored by W.-D. Ruan in 1999, but was recently revisited in R. Guadagni's 2017 University of Texas Ph.D. thesis.
}

\section{Looking Ahead}

From this symplectic geometer's perspective, the most important task ahead is to fill the gaps in the picture just outlined-precisely how to construct Lagrangian fibrations with singularities from degenerations, and then, crucially, how to construct their analytic mirrors. The chief difficulty is with Floer theory for singular Lagrangians. The GrossSiebert program provides an algebro-geometric solution, at the cost of losing the direct connection to symplectic topology and the natural construction of HMS as a Fourier transform. I hope and suspect that Gross-Siebert's work will be precisely linked to symplectic topology, perhaps even in the absence of a full understanding of the singular Lagrangians, and that a proof of HMS, valid in vastly more generality than we can currently manage, will thereby emerge.

I especially look forward to the weaving together of different threads of mirror symmetry, integrating the symplectic-analytic-algebraic picture with the Riemannian geometry of special Lagrangians; and the topological field theory of the A- and B-models with rigorous approaches to a quantum field theory on $\check{X}[3,14]$. In this account I have not even touched on mirror symmetry for Fano manifolds-which is just as remarkable as for $\mathrm{CY}$ manifolds-nor on wall-crossing, applications of mirror symmetry in symplectic topology, or connections to the Langlands program. For mathematicians fascinated by hidden connections, mirror symmetry is a dazzling phenomenon.

\section{References}

[1] Abouzaid M, Family Floer cohomology and mirror symmetry. Proceedings of the International Congress of Mathematicians-Seoul 2014. II, 813-836, Kyung Moon Sa, Seoul, 2014. MR3728639

[2] Abouzaid M, Homological mirror symmetry without corrections. ArXiv:1703.07898

[3] Bershadsky M, Cecotti S, Ooguri H, Vafa C, KodairaSpencer theory of gravity and exact results for quantum string amplitudes. Comm. Math. Phys. 165 (1994), no. 2, 311-427. MR1301851

[4] Candelas P, de la Ossa X, Green P, Parkes L, An exactly soluble superconformal theory from a mirror pair of CalabiYau manifolds. Particles, strings and cosmology (Boston, MA, 1991), 667-680, World Sci. Publ., River Edge, NJ, 1992. MR1399815

[5] Costello K, Topological conformal field theories and Calabi-Yau categories. Adv. Math. 210 (2007), no.1, 165214. MR2298823

[6] Fukaya K, Cyclic symmetry and adic convergence in Lagrangian Floer theory. Kyoto J. Math. 50 (2010), no. 3, 521590. MR2723862

[7] Givental A, A mirror theorem for toric complete intersections. Topological Field Theory, Primitive Forms and Related 
FEATURED TITLES FROM THE

\section{EUROPEAN MATHEMATICAL SOCIETY}

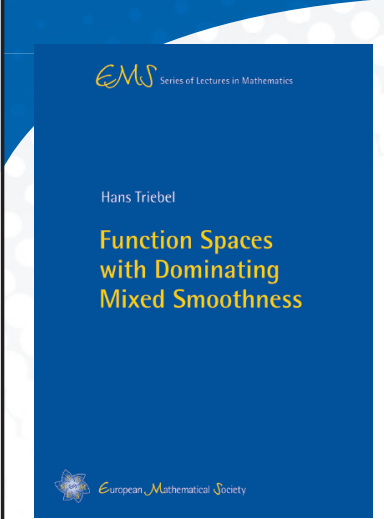

Function Spaces with Dominating Mixed Smoothness

Hans Triebel, University of Jena, Germany

These notes are addressed to graduate students and mathematicians who have a working knowledge of basic elements of the theory of function spaces, especially of Besov-Sobolev type. In particular, the book will be of interest to researchers dealing with approximation theory, numerical integration, and discrepancy.

EMS Series of Lectures in Mathematics, Volume 30; 2019; 210 pages; Softcover; ISBN: 978-3-03719-195-8; List US\$42; AMS members US\$33.60; Order code EMSSERLEC/30

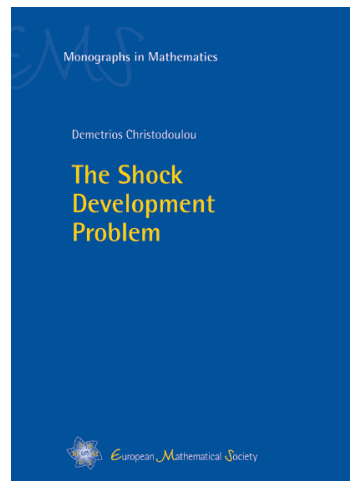

\section{The Shock Development Problem}

Demetrios Christodoulou, Eidgen Technische Hochschule, Zurich, Switzerland

This monograph addresses the problem of the development of shocks in the context of the Eulerian equations of the mechanics of compressible fluids. The mathematical problem is that of an initial-boundary value problem for a nonlinear hyperbolic system of partial differential equations with a free boundary and singular initial conditions.

EMS Monographs in Mathematics, Volume 8; 2019; 932 pages; Hardcover; ISBN: 978-3-03719-192-7; List US\$148; AMS members US\$118.40; Order code EMSMONO/8

\section{Explore more titles at bookstore.ams.org.}

Publications of the European Mathematical Society (EMS). Distributed within the Americas by the American Mathematical Society.

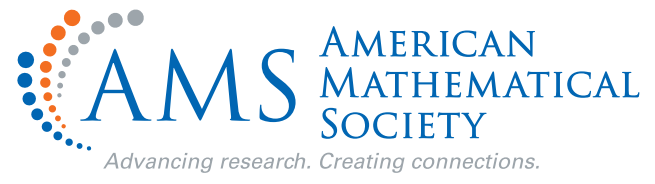

Topics, Progress in Mathematics, 160 (1998), Birkhäuser Boston, MA MR1653024

[8] Ganatra S, Perutz T, Sheridan N, Mirror symmetry: from categories to curve counts, arXiv: 1510.03839

[9] Greene B, Plesser M, Duality in Calabi-Yau moduli space. Nuclear Phys. B 338 (1990), no. 1, 15-37. MR1059831

[10] Gross M, Siebert B, An invitation to toric degenerations. Surveys in differential geometry. Volume XVI. Geometry of special holonomy and related topics, 43-78, Surv. Differ. Geom., 16, Int. Press, Somerville, MA, 2011. MR2893676

[11] Kontsevich M, Homological algebra of mirror symmetry. Proceedings of the International Congress of Mathematicians, Vol. 1, 2 (Zürich, 1994), 120-139, Birkhäuser, Basel, 1995. MR1403918

[12] Kontsevich M, Soibelman Y, Affine structures and nonArchimedean analytic spaces. The unity of mathematics, 321385, Progr. Math., 244, Birkhäuser Boston, Boston, MA, 2006. MR2181810

[13] Lerche W, Vafa C, Warner N, Chiral rings in $N=2$ superconformal theories. Nuclear Phys. B 324 (1989), no. 2, 427-474. MR1025424

[14] Li Q, Li S, On the B-twisted topological sigma model and Calabi-Yau geometry. J. Differential Geom. 102 (2016), no. 3, 409-484. MR3466804

[15] Mukai S, On the moduli space of bundles on K3 surfaces. I. Vector bundles on algebraic varieties (Bombay, 1984), $341-$ 413, Tata Inst. Fund. Res. Stud. Math., 11, Tata Inst. Fund. Res., Bombay, 1987. MR893604

[16] Sheridan N, Homological mirror symmetry for CalabiYau hypersurfaces in projective space, Invent. Math. 199 (2015), no. 1, 1-186. MR3294958

[17] Strominger A, Yau S-T, Zaslow E, Mirror symmetry is T-duality, Nuclear Phys. B 479 (1996), no. 1-2, 243-259. MR1429831

[18] Tate J, Rigid analytic spaces. Invent. Math. 12 (1971), 257-289. MR0306196

[19] Witten E, Mirror manifolds and topological field theory. Essays on mirror manifolds, 120-158, Int. Press, Hong Kong, 1992. MR1191422

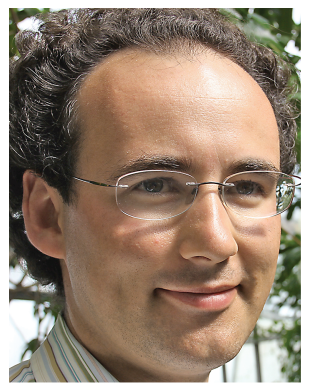

Timothy Perutz

Credits

All images are courtesy of Timothy Perutz. 